\title{
Analysis of the contribution to type 2 diabetes susceptibility of sequence variation in the gene encoding stearoyl-CoA desaturase, a key regulator of lipid and carbohydrate metabolism
}

\author{
C. F. Liew ${ }^{1,2,3}$ • C. J. Groves ${ }^{1,2}$ - S. Wiltshire ${ }^{2}$ E. Zeggini ${ }^{2}$ T. M. Frayling ${ }^{4}$ K. R. Owen ${ }^{4}$ M. Walker ${ }^{5}$ \\ G. A. Hitman ${ }^{6}$ J. C. Levy ${ }^{1}$ S. O' Rahilly ${ }^{7}$ A. T. Hattersley ${ }^{4}$ D. G. Johnston ${ }^{8}$ - M. I. McCarthy ${ }^{1,2}$ \\ ${ }^{1}$ Oxford Centre for Diabetes, Endocrinology and Metabolism, Churchill Hospital, Oxford, UK \\ ${ }^{2}$ Wellcome Trust Centre for Human Genetics, Oxford, UK \\ ${ }^{3}$ Department of Medicine, National University Hospital, Singapore \\ ${ }^{4}$ Centre for Molecular Genetics, Peninsula Medical School, Exeter, UK \\ 5 School of Clinical Medical Sciences, University of Newcastle, Newcastle, UK \\ ${ }^{6}$ Department of Diabetes and Metabolic Medicine, Bart's and The London Queen Mary's School of Medicine and Dentistry, \\ London, UK \\ ${ }^{7}$ Departments of Medicine and Clinical Biochemistry, Addenbrooke's Hospital, Cambridge, UK \\ ${ }^{8}$ Section of Endocrinology and Metabolic Medicine, Imperial College Faculty of Medicine, St. Mary’s Hospital, London, UK
}

\begin{abstract}
Aims/hypothesis. Stearoyl-CoA desaturase (SCD) is emerging as a key regulator of lipid and carbohydrate metabolism. Scd-null mice display a beneficial metabolic phenotype characterised by resistance to obesity, diabetes and hyperlipidaemia. The human homologue, $S C D$, maps to a region of chromosome 10 linked to type 2 diabetes, and SCD activity correlates with insulin sensitivity. Given this strong positional and biological candidacy, the present study sought to establish whether sequence variation in $S C D$ influences susceptibility to type 2 diabetes and related traits.

Methods. The SCD gene was resequenced in 23 diabetic subjects. Six variants within coding and adjacent sequence, including a non-synonymous SNP in exon 5 (M224L), were selected for genotyping in a primary set of 608 diabetic subjects and 600 control subjects. Results. There was no association (at the allele, genotype or haplotype level) with type 2 diabetes, although
\end{abstract}

genotype frequencies at the $+14301 \mathrm{~A}>\mathrm{C}$ SNP in the $3^{\prime}$ untranslated region showed borderline association ( $p \sim 0.06$ ) when evidence for linkage was taken into account. However, replication studies (350 young-onset diabetic patients; 747 controls) failed to confirm any relationship with diabetes for this variant. No significant associations were seen for diabetes-related traits including BMI and waist-to-hip ratio.

Conclusions/interpretation. The present study, the first reported analysis of this gene, indicates that the $S C D$ variants typed do not explain chromosome-10-encoded susceptibility to type 2 diabetes. Although this study provided no evidence that $S C D$ sequence variation influences diabetes susceptibility or related traits, SCD remains a major target for pharmaceutical and/or environmental manipulation.

Keywords Association - Chromosome 10 - Linkage disequilibrium $\cdot$ Lipids $\cdot$ Metabolism $\cdot$ Stearoyl-CoA desaturase.
Received: 29 July 2004 / Accepted: 2 October 2004

Published online: 15 December 2004

(C) Springer-Verlag 2004

M. I. McCarthy (๘)

Oxford Centre for Diabetes, Endocrinology and Metabolism,

Churchill Hospital, Old Road, Oxford, OX3 7LJ, UK

E-mail: mark.mccarthy@drl.ox.ac.uk

Tel.: +44-1865-857298, Fax: +44-1865-857299

Abbreviations: DIF, Diabetes in Families study · ECACC, European Collection of Cell Cultures · EFS, Exeter Family Study · GIST, Genotype-IBD Sharing Test · GRR, Genotype Relative Risk · MUFA, monounsaturated fatty acids · NPL, non-parametric linkage $\cdot$ SCD, stearoyl-CoA desaturase $\cdot$ SFA, saturated fatty acids - SNP, single nucleotide polymorphism * UTR, untranslated region

\section{Introduction}

The shift from an exclusively glucocentric view of type 2 diabetes pathogenesis to one which emphasises the importance of defects in lipid metabolism and adipocyte function has generated important insights into the pathogenesis of this condition [1,2]. Support for this lipocentric view has been driven by increasing awareness of the diverse and adverse metabolic consequences of raised fatty acid levels both on insulin action and secretion $[3,4]$, and by the unmasking of the fat cell as a potent endocrine organ [5]. This change of focus has already led to the identification of 
several susceptibility genes influencing type 2 diabetes and related traits. For example, variants in PPARG2 (encoding peroxisomal proliferator-activated receptor gamma) and in $C d 36$ (encoding the fatty acid translocase, CD36) have been implicated in the development of type 2 diabetes and insulin resistance in humans and rodents respectively [6,7]. As a result, there is growing interest in other key regulators of intermediary lipid metabolism and an expectation that such studies will reveal additional genes conferring type 2 diabetes susceptibility, provide clues to fundamental pathophysiological processes, and inform development of novel therapeutic agents. Stearoyl-CoA desaturase (SCD: EC reference 1.1499.5) is one such key regulator, and the aim of the present study was to determine whether sequence variation in the gene encoding SCD (SCD, Locuslink reference 6319) is associated with type 2 diabetes risk and/or related intermediate traits.

Several lines of evidence establish $S C D$ as a strong biological and positional candidate.

Firstly, SCD is the rate-limiting enzyme in the cellular biosynthesis of monounsaturated fatty acids (MUFA) from saturated fatty acid (SFA) substrates (mainly palmitoyl and stearoyl) [8]. As a result of substrate channelling, MUFAs generated by SCD, as opposed to those derived directly from dietary sources, represent the main substrates for synthesis of the triglycerides, cholesterol esters and phospholipids that subserve a wide range of cellular and metabolic functions $[8,9]$. Consistent with a role as a key point of metabolic control, SCD activity is tightly regulated [10], being decreased by polyunsaturated fatty acids [11] and leptin [12], but increased by SFA and cholesterol [11].

Secondly, mice deficient in SCD (both the natural asebia and the Scdl knock-out mouse) are characterised by a metabolic phenotype that, in many respects, reflects "protection" against the metabolic syndrome. Compared to wild-type mice, SCD-deficient mice have low circulating levels of lipids [13]; reduced body fat, despite hyperphagia [12]; resistance to weight gain and to development of liver steatosis when transferred to a high-fat diet [14]; increased metabolic rate and fat oxidation [12]; marked resistance to obesity when crossed with the leptin-deficient $o b / o b$ mouse [12]; and improved insulin sensitivity and glucose tolerance $[8,14]$. Recent data have elucidated the mechanisms responsible for this favourable metabolic profile. Scd-deficiency activates AMPkinase, leading to phosphorylation and inhibition of acetyl-CoA carboxylase activity [15]. The resulting reduction of malonyl CoA de-represses the mitochondrial shuttle carnitine palmitoyltransferase 1, promoting fatty acid import into mitochondria [15].

Thirdly, human physiological and biochemical studies, although limited to date, have demonstrated a positive correlation between measures of SCD activity (inferred from the ratio of circulating $\mathrm{C}_{18: 1} / \mathrm{C}_{18: 0}$ fatty acids) and circulating triglyceride levels (as a measure of insulin resistance) [16, 17].

Finally, $S C D$, the human homologue of murine Scd1, maps to the long arm of chromosome 10 (10q23-24). Evidence for linkage to type 2 diabetes at chromosome 10q has been reported in several studies $[18,19,20,21,22]$, including the UK Warren 2 study, where a LOD of 1.99 was found at D10S1765 (approximately $12 \mathrm{Mb}$ pter to $S C D$ on the current genome assembly [NCBI version 34, accessed at www.ensembl.org, 23 September, 2004]). This is the first study to test the hypothesis that $S C D$ variation contributes to type 2 diabetes susceptibility.

\section{Materials and methods}

Subjects. This study included analysis of two case and two control groups: clinical characteristics are provided in Table 1. In the primary case-control analysis, we compared unrelated probands with type 2 diabetes $(n=608)$ from the Diabetes UK Warren 2 sibpair repository (Warren 2 probands) [18] with a control panel from two sources: 348 random UK population control samples from the European Collection of Cell Cultures (ECACC; Salisbury, UK) and 252 UK subjects ascertained (as spouse controls) from the Diabetes in Families study (DIF). For extension and replication studies, additional case samples were obtained by combining two sets of young-onset type 2 diabetic subjects $(n=350)$ with almost identical clinical characteristics: (i) offspring from parent-offspring trios $(n=147)$ ascertained for type 2 diabetes [23]; and (ii) young-onset ( $<45$ years) type 2 diabetic subjects $(n=203)$ [24, 25]. These cases were compared to 747 normoglycaemic parents from a consecutive birth cohort (the Exeter Family Study [EFS]) [24]. All case samples were therefore strongly selected for inherited type 2 diabetes on the basis of early disease onset and/or positive family history. Other types of diabetes were excluded using a combination of clinical, immunological and genetic criteria as previously described $[18,23,25]$. Confirmation of glycaemic status in the control populations was limited to fasting plasma glucose measures for the EFS samples. All subjects are of exclusively British/Irish origin. Informed consent was obtained from all subjects, and all studies were carried out in accordance with the principles of the Declaration of Helsinki (2000).

$S C D$ resequencing and genotyping. For resequencing, primers were designed to amplify the six exons of $S C D$, their immediate flanking intronic sequence, the extensive $3^{\prime} \mathrm{UTR}$ (untranslated region; $3.8 \mathrm{~Kb}$ ) and $1000 \mathrm{bp}$ of 5' "promoter" sequence. The 16 amplicons that resulted were screened for variation by sequencing 23 probands with type 2 diabetes from the Diabetes UK Warren 2 repository. This number of samples has greater than $90 \%$ power to detect a disease-associated variant that has a greater than 5\% frequency amongst cases ascertained from multiplex families. PCR products were bi-directionally sequenced using standard dye terminator chemistry (BIG DYE v 3.0, Applied Biosystems, Foster City, Calif., USA) on an ABI3700 capillary sequencer. Sequences were assembled and aligned using Phred and Phrap [26, 27]. Potential polymorphisms identified using Polyphred [28] were confirmed where possible by cross referencing to public databases. A subset of the variants identified was selected for genotyping based on al- 
Table 1. Subject group details

\begin{tabular}{|c|c|c|c|}
\hline & $\begin{array}{l}\text { W2P } \\
\text { Case }\end{array}$ & $\begin{array}{l}\text { ECACC } \\
\text { Control }\end{array}$ & $\begin{array}{l}\text { DIF } \\
\text { Control }\end{array}$ \\
\hline Number & 608 & 348 & 252 \\
\hline Age at diagnosis ${ }^{\mathrm{a}}$ / examination ${ }^{\mathrm{b}}$ (years) & $55.3(8.5)^{\mathrm{a}}$ & $38.6(8.1)^{\mathrm{b}, \mathrm{c}}$ & $55.0(18.4)^{\mathrm{b}}$ \\
\hline BMI (male) $\left(\mathrm{kg} / \mathrm{m}^{2}\right)$ & $27.7(4.1)$ & - & $25.0(4.6)$ \\
\hline BMI (female) $\left(\mathrm{kg} / \mathrm{m}^{2}\right)$ & $30.2(5.8)$ & - & $24.6(4.2)$ \\
\hline \multirow{2}{*}{ WHR (female) } & Y2TD & & EFS \\
\hline & Case & & Control \\
\hline Number & 350 & & 747 \\
\hline WHR (male) & $0.97(0.06)$ & & $0.88(0.06)$ \\
\hline WHR (female) & $0.89(0.08)$ & & $-d$ \\
\hline
\end{tabular}

Continuous data are means (SD). Age was recorded at a diagnosis for the cases and $a^{b}{ }^{b}$ study commencement for the controls. c Age information was available for only $34 \%$ of subjects in this group. ${ }^{\mathrm{d}} \mathrm{BMI}$ in females was measured during pregnan- cy: meaningful measures of WHR were not available. W2P, Warren 2 probands; ECACC, European Collection of Cell Cultures; DIF, Diabetes in Families study; Y2TD, young-onset type 2 diabetes; EFS, Exeter Family Study

Table 2. SNPs within $S C D$ identified through resequencing

\begin{tabular}{|c|c|c|c|c|c|c|c|}
\hline SNP & Location & $\begin{array}{l}\text { Peptide } \\
\text { change }\end{array}$ & dbSNP ID & ${ }^{\mathrm{a} G e n o m i c ~ d e s i g n a t i o n ~}$ & $\begin{array}{l}\text { b Relative to } \\
\text { ATG }(+1)\end{array}$ & $\begin{array}{l}\text { cNCBI34 } \\
\text { Position }\end{array}$ & SNP Assay \\
\hline SNP1 & Promoter & - & rs2275656 & g.al139819.8:67634G $>C$ & -1057 & 101770792 & Not typed \\
\hline SNP3 & Promoter & - & rs670213 & g.al139819.8:67796T>C & -895 & 101770954 & Amplifluor \\
\hline SNP4 & Intron 4 & - & rs3071 & g.al139819.8:75892A >C & +7202 & 101779050 & PCR-RFLP (DdeI) \\
\hline SNP5 & Exon 5 & M224L & rs 11598233 & g.al139819.8:77740A $>C$ & +9050 & 101780898 & 4 primer ARMS \\
\hline SNP8 & 3' UTR & - & - & g.al139819.8:82991A $>C$ & +14301 & 101786149 & PCR-RFLP $(H p y C H 4 I V)$ \\
\hline SNP9 & $3^{\prime} \mathrm{UTR}$ & - & rs3978768 & g.al139819.8:83008A $>G$ & +14318 & 101786166 & PCR-RFLP (Tsp509I) \\
\hline SNP10 & $3^{\prime}$ UTR & - & - & g.al139819.8:83245T>G & +14555 & 101786403 & Not typed \\
\hline
\end{tabular}

Identification of the 10 variants detected in the human SCD gene. The position of each SNP is recorded with reference to: a the relevant BAC clone assembly (AL139819.8); ${ }^{\mathrm{b}}$ the transla- tion initiation site (ATG=+1); and c the NCBI34 genome assembly. ARMS, amplification refractory mutation system lele frequencies and linkage disequilibrium relationships, supported by statistical methods (LDSelect [29]). Selected variants were genotyped using a combination of (i) gel-based PCR-RFLP assays incorporating obligate restriction sites as an internal digest control; (ii) tetra primer amplification refractory mutation system PCR [30]); and (iii) fluorescent probe-based Amplifluor methods [31] (Table 2). Primer sequences, amplification conditions and additional genotyping details are available from the authors. Based on extensive duplicate genotyping and analysis of haplotype patterns, we estimate an overall genotyping error rate of less than $0.5 \%$.

Statistical analysis. SNPs were tested separately in cases and controls for deviation from Hardy-Weinberg equilibrium using HelixTree Genetics Analysis Software (Golden Helix, Boze- man, Mont., USA) [32]. Genotype and allele frequency distributions were compared by contingency table methods, and genotype trend comparisons carried out using the Kruskal-Wallis test and the generalised Cochran-Mantel-Haenszel statistic. As necessary, exact $p$ values for these tests were obtained using StatXact 6 (Cytel Software, Cambridge, Mass., USA). Separately ascertained subject groups were combined for analysis only after the appropriate homogeneity tests had been applied. Measures of pairwise linkage disequilibrium $\left(r^{2}\right.$ and $\left.\mathrm{D}^{\prime}\right)$ were estimated using the expectationmaximisation algorithm implemented by the LDmax function in GOLD [32]. Haplotype patterns were estimated by maximum-likelihood methods (SNPHAP, http://www-gene. cimr.cam.ac.uk/clayton/software, accessed 23 September, 2004) and haplotype frequency distributions compared by like- 
lihood-ratio testing. Significance was determined by permutation (1000 replicates). Two complementary approaches were used to assess evidence for linkage partitioning. First, Warren 2 probands were subdivided into linked (family non-parametric linkage [NPL] score $\geq 0$ at peak of 10q linkage [D10S1765]) and unlinked (family NPL score $<0$ ), with genotype and allele distributions compared as described above. Second, the Genotype-IBD Sharing Test (GIST) [33] was used to assess whether variation at any of the SCD SNPs accounted for any part of the chromosome 10 linkage signal under dominant, recessive and additive models. Analysis was performed using the modelweighted variables and the family NPL score (generated by ALLEGRO [34]) as input values. The effect of variation at individual $S C D$ SNPs on relevant intermediate quantitative traits was assessed by ANOVA using appropriately transformed variables (SPSS12.0 SPSS, Chicago, Ill., USA). For haplotype effects, we used haplotype trend regression, as implemented in HelixTree Genetics Analysis Software (Golden Helix) [35]. For the primary case-control analysis, a $p$ value of less than 0.05 was taken as the level for reporting significance; for the intermediate trait analyses, we adopted a more stringent $p$ value $(<0.01)$ to account for the increased type 1 error rate associated with testing of multiple phenotypes.

\section{Results}

$S C D$ resequencing detected ten variants (all single nucleotide polymorphisms [SNPs]) (Table 2). For convenience, these are referred to as SNP1 to SNP10. Six of these corresponded to existing entries in the public SNP databases, but four were novel (SNPs 6, 7, 8 and 10). Two of the variants lay in coding sequence, SNP6 being synonymous (Y308Y) and SNP5 non-synonymous (M224L). The remaining SNPs were non-coding and located either in the promoter region, introns or extended 3' UTR. SNPs with a minor allele frequency below 5\% (SNP6 and SNP7) were not considered further. SNP1 and SNP10 were found to be in very tight linkage disequilibrium with SNP3 $\left(r^{2}=0.91, \mathrm{D}^{\prime}=1\right)$ and SNP8 $\left(r^{2}=1, \mathrm{D}^{\prime}=1\right)$ respectively, and were therefore excluded from further genotyping. Based on the haplotypes determined from our resequencing experiments, the remaining six SNPs $(2,3,4,5,8$ and 9) captured the majority ( $>98 \%$ ) of the haplotype diversity in the samples sequenced, and were therefore selected for genotyping in the larger case-control samples. The haplotype diversity of this region identified by our resequencing is confirmed by genotype data currently available from the HapMap (www. hapmap.org, release 8, accessed June 2004).

All SNPs typed were in Hardy-Weinberg equilibrium except SNP2 ( $p=0.014$ in the control sample). This deviation was considered to reflect stochastic variation as there was complete correspondence between duplicate samples and also between genotypes and resequencing data. In addition, there was no indication of mistyping when haplotypes were inspected (i.e. appearance of novel haplotypes on particular plates). Analysis of the control sample demonstrated moderate linkage disequilibrium across the gene with all pair-
Table 3. Pairwise linkage disequilibrium in control samples

\begin{tabular}{lllllll}
\hline & SNP2 & SNP3 & SNP4 & SNP5 & SNP8 & SNP9 \\
\hline SNP2 & & 0.55 & 0.43 & 0.38 & 0.07 & 0.24 \\
SNP3 & 0.99 & & 0.21 & 0.67 & 0.11 & 0.44 \\
SNP4 & 0.81 & 0.76 & & 0.28 & 0.05 & 0.07 \\
SNP5 & 0.94 & 0.95 & 0.99 & & 0.09 & 0.44 \\
SNP8 & 1.00 & 0.97 & 1.00 & 1.00 & & 0.06 \\
SNP9 & 0.90 & 0.92 & 0.59 & 0.79 & 1.00 & \\
& $\mathrm{D}^{\prime}$ & & & & & \\
\hline
\end{tabular}

Measures of linkage disequilibrium ( $\mathrm{D}^{\prime}$ and $r^{2}$ ) calculated in the primary control samples (calculated using expectation-maximisation methods [32])

wise $\mathrm{D}^{\prime}$ values being greater than 0.59 , and pairwise $r^{2}$ measures ranging from 0.05 to 0.67 (Table 3). Application of variant selection tools (LDSelect) to the genotypes subsequently obtained in the control samples failed to identify any more parsimonious subset of SNPs able to tag the variation at this locus.

Genotype frequencies for the Warren 2 probands (cases) and controls are shown in Table 4. No significant association was detected between the cases and controls in the primary sample when genotype and allele frequencies were compared, nor was there a difference in the haplotype distribution as assessed by permutation test (1000 replicates; $p=0.43$ ) (Table 5). To assess whether genotype variation had an effect on the linkage signal at that locus, the case sample was subdivided according to the family NPL score at the peak of linkage on chromosome 10q (D10S1765) (see Methods). There was no significant difference in genotype distribution between the linked and unlinked type 2 diabetic probands at any of the SNPs. However, the comparison at SNP8 was of borderline significance $(p=0.059)$, reflecting a decrease in the frequency of the AA genotype in the linked families $(79.1 \%$ vs $86.3 \%$ ). GIST analysis found no evidence that the $S C D$ SNPs (including SNP8) contributed to the 10q linkage signal (data not shown).

Given the weak evidence for a linkage-partitioning effect at SNP8, genotyping of this variant was extended to the replicate sample set (350 young-onset type 2 diabetic subjects; 747 controls). In this set there was no suggestion of association with type 2 diabetes $(p=0.50)$. Analysis of all SNP8 genotype data (using the Cochran-Mantel-Haenszel test, for ordered genotypes) confirmed that there was no overall association (exact $p=0.78$ ).

Relationships between $S C D$ sequence variation and relevant intermediate traits (BMI, waist-to-hip ratio) were sought in the Warren 2 probands with diabetes, and in the DIF controls (trait information was not available for the ECACC control subjects). Investigation of these traits provided no evidence for singlepoint or haplotypic associations when case and control individuals were analysed separately $(p>0.01)$. 


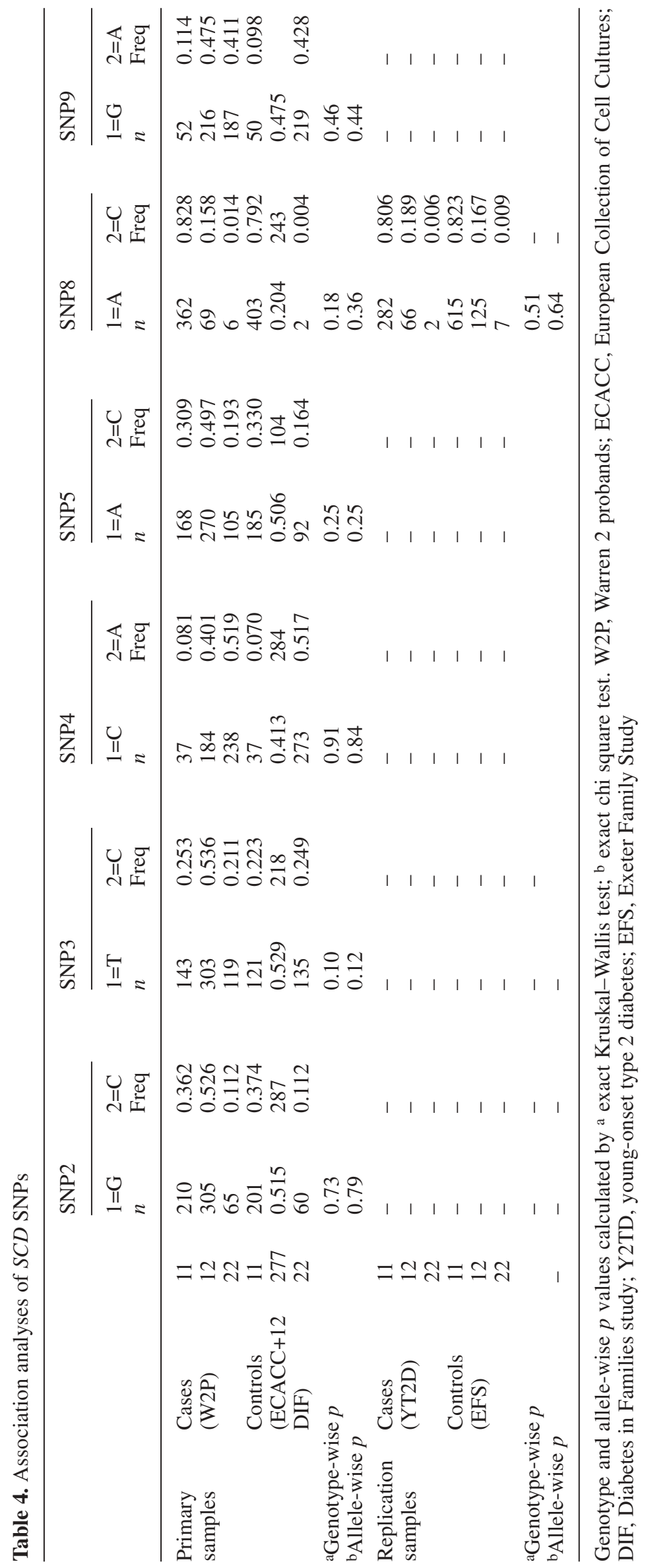


Table 5. Haplotype frequencies in cases and controls

\begin{tabular}{lll}
\hline $\begin{array}{l}\text { SCD haplotype } \\
2-3-4-5-8-9\end{array}$ & $\begin{array}{l}\text { Cases } \\
\text { (W2P) }\end{array}$ & $\begin{array}{l}\text { Controls } \\
\text { (ECACC+DIF) }\end{array}$ \\
\hline 112211 & 0.288 & 0.284 \\
221112 & 0.230 & 0.242 \\
112212 & 0.127 & 0.117 \\
222112 & 0.120 & 0.108 \\
122122 & 0.087 & 0.105 \\
112112 & 0.051 & 0.050 \\
111111 & 0.042 & 0.032 \\
122112 & 0.020 & 0.034 \\
222211 & 0.011 & 0.006 \\
Others & 0.026 & 0.022 \\
$P$ & 0.429 & \\
\hline
\end{tabular}

Six SNP haplotypes are shown in location order. Haplotype frequencies were estimated and compared using maximumlikelihood methods with significance determined using permutation (1000 replicates). W2P, Warren 2 probands; ECACC, European Collection of Cell Cultures; DIF, Diabetes in Families study

\section{Discussion}

To our knowledge, this is the first detailed study of the association between $S C D$ variation and type 2 diabetes. We have undertaken a comprehensive survey for variation in the exons, adjacent intronic sequences, as well as $1 \mathrm{~kb}$ of upstream sequence (covering known promoter elements [36]) and the extensive $3^{\prime}$ UTR. A subset of those variants, including a non-synonymous variant in exon 5, were then typed in a sample of type 2 diabetic subjects (enriched for diabetes-susceptibility genes by virtue of a strong family history) and ethnically matched controls. No convincing evidence of association was detected at the level of the allele, genotype or haplotype. The suggestion that variation at SNP8 might be correlated to the family-wise evidence for linkage to chromosome 10 was not substantiated by further genotyping in the replicate sample. In addition, secondary analyses looking at diabetes-related continuous traits (such as BMI and waist-to-hip ratio) failed to detect any convincing evidence for association.

The clear conclusion from these data is that $S C D$ variation does not contribute to type 2 diabetes susceptibility in these samples. As in any association study, wider interpretation of these findings needs to take account of several crucial factors.

The first is that, whilst the present study focused on those elements of the gene most likely to be relevant to $S C D$ function and/or expression, it did not directly test all variants that might conceivably influence these outcomes. Through our own resequencing, combined with a survey of the publicly available data, we are confident that we have detected all common variants within the coding, promoter and UTR regions. The single non-synonymous coding change (SNP5:
M224L) represents a conservative substitution within the transmembrane domain, though it is notable that the L224 residue is conserved across multiple mammalian species.

The available HapMap data confirm our own finding that this gene is characterised by appreciable haplotype diversity, but that the six selected SNPs capture the majority of that variation. We therefore infer that the selected SNPs had reasonable power to detect associations, if common variants in adjacent sequence were influencing type 2 diabetes susceptibility [37]. However, the current study was not designed to detect susceptibility effects attributable to rare variants. Nor would it be capable of detecting susceptibility effects due to variants lying at some distance from the "core" gene elements (exons, promoter, UTRs). Homology mapping of mammalian (mouse, rat, human) genome sequence (using ECR browser, http://ecrbrowser.dcode.org, accessed 23 September 2004 [38]) around the $S C D$ gene reveals several conserved noncoding sequences that are strong candidates for a functional role, and which therefore merit efforts to detect and type additional sequence variation.

The second issue is sample size. The power of the primary case-control resource used in the present study is enhanced by the fact that the cases were selected for strong family history. This manoeuvre has a substantial, beneficial effect on study power [39, 40]. Even allowing for this, the primary case-control study only has power to detect a relatively large effect size. For example, under a multiplicative model, the current study (allowing for selected case ascertainment) has around $90 \%$ power to detect (at a threshold of $p<0.05$ ) a susceptibility variant with an effect size (as measured in terms of the penetrance ratio, or genotypic relative risk [GRR]) of 1.2 and control allele frequency greater than 30\%. For larger effect sizes (GRR 1.3), similar power is obtained for less common alleles (control allele frequency $>10 \%$ ). In the present study, we adopted a relaxed $p$ value for the primary case-control study, in the expectation that any associations showing weak or borderline association with type 2 diabetes (as in the case of SNP8) would be examined further in the replication samples. Clearly, the present study cannot exclude the possibility that the variants typed (or others with which they are in linkage disequilibrium) do indeed influence type 2 diabetes susceptibility, but have effect sizes too small to have been detected in these samples. It is important to note however, that the present study has more than adequate power to reject the specific hypothesis that the $S C D$ variants typed are solely responsible for the linkage signal in this region detected in the UK genome scan [18], since this hypothesis implies effect sizes very much greater than those considered in the power calculations above.

Finally, it is important to recognise that the failure of the present study to detect significant associations 
with type 2 diabetes susceptibility does not exclude the possibility that these (or other $S C D$ ) variants influence intermediate traits relevant to the pathogenesis of type 2 diabetes. It is possible that type 2 diabetes represents too complex and too distal a phenotype to be optimal for detection of phenotypic correlates of functional variation within the gene. The lack of any significant associations with BMI and waist-to-hip ratio to some extent argues against this possibility. However, future studies will need to consider these variants in relation to more proximal intermediate traits including direct (enzymatic activity in microsomal fractions from adipose tissue biopsies) and indirect (18:1/18:0 ratios in lipid fractions) measures of SCD activity.

The identification of genetic variants that cause or influence susceptibility to diabetes and/or related traits represents a powerful tool for demonstrating the functional role of a given gene and its product in human metabolic function. The failure to detect such an association in the present study means that such validation is not yet available. However, physiological studies of SCD function in humans are consistent with the important role in the maintenance of normal metabolic homeostasis that has been clearly demonstrated in the mouse. As such, SCD remains an interesting target for pharmaceutical intervention, capable, in principle of simultaneously reversing several components of the metabolic syndrome.

Acknowledgements. C.F. Liew and C.J. Groves contributed equally to this article. This work was supported by Diabetes UK, who were also responsible for funding the collection of many of the samples used. We are grateful to Golden Helix for making the HelixTree software package available to us and to Johnny Holme and Phil Robinson at KBiosciences (Hoddesdon, Herts, UK) for assistance with assay design and genotyping. A.T. Hattersley is a Wellcome Trust Research Leave Fellow.

\section{References}

1. Shafrir E, Raz I (2003) Diabetes: mellitus or lipidus? Diabetologia 46:433-440

2. Schrauwen P, Hesselink MKC (2004) Oxidative capacity, lipotoxicity, and mitochondrial damage in type 2 diabetes. Diabetes 53:1412-1417

3. Yki-Jarvinen H (2002) Ectopic fat accumulation: an important cause of insulin resistance in humans. J R Soc Med 95 [Suppl 42]:39-45

4. Winzell MS, Svensson H, Enerbäck S et al. (2003) Pancreatic beta-cell lipotoxicity induced by overexpression of hormone-sensitive lipase. Diabetes 52:2057-2065

5. Yamauchi T, Kamon J, Waki H et al. (2001) The fatderived hormone adiponectin reverses insulin resistance associated with both lipoatrophy and obesity. Nat Med 7:941-946

6. Altshuler D, Hirschhorn JN, Klannemark M et al. (2000) The common PPARgamma Pro12Ala polymorphism is associated with decreased risk of type 2 diabetes. Nat Genet 26:76-80

7. Aitman TJ, Glazier AM, Wallace CA et al. (1999) Identification of $\mathrm{Cd} 36$ (Fat) as an insulin-resistance gene causing defective fatty acid and glucose metabolism in hypertensive rats. Nat Genet 21:76-83

8. Ntambi JM, Miyazaki M (2003) Recent insights into stearoyl-CoA desaturase-1. Curr Opin Lipidol 14:255-261

9. Ntambi JM, Miyazaki M (2004) Regulation of stearoylCoA desaturases and role in metabolism. Prog Lipid Res 43:91-104

10. Ntambi JM (1999) Regulation of stearoyl-CoA desaturase by polyunsaturated fatty acids and cholesterol. J Lipid Res 40:1549-1558

11. Jones BH, Maher MA, Banz WJ et al. (1996) Adipose tissue stearoyl-CoA desaturase mRNA is increased by obesity and decreased by polyunsaturated fatty acids. Am J Physiol 271:E44-E49

12. Cohen P, Miyazaki M, Socci ND et al. (2002) Role for stearoyl-CoA desaturase-1 in leptin-mediated weight loss. Science 297:240-243

13. Miyazaki M, Kim YC, Ntambi JM (2001) A lipogenic diet in mice with a disruption of the stearoyl-CoA desaturase 1 gene reveals a stringent requirement of endogenous monounsaturated fatty acids for triglyceride synthesis. J Lipid Res 42:1018-1024

14. Ntambi JM, Miyazaki M, Stoehr JP et al. (2002) Loss of stearoyl-CoA desaturase-1 function protects mice against adiposity. Proc Natl Acad Sci USA 99:11482-11486

15. Dobrzyn P, Dobrzyn A, Miyazaki M et al. (2004) StearoylCoA desaturase 1 deficiency increases fatty acid oxidation by activating AMP-activated protein kinase in liver. Proc Natl Acad Sci USA 101:6409-6414

16. Attie AD, Krauss RM, Gray-Keller MP et al. (2002) Relationship between stearoyl-CoA desaturase activity and plasma triglycerides in human and mouse hypertriglyceridemia. J Lipid Res 43:1899-1907

17. Shiwaku K, Hashimoto M, Kitajima K et al. (2004) Triglyceride levels are ethnic-specifically associated with an index of stearoyl-CoA desaturase activity and n-3 PUFA levels in Asians. J Lipid Res 45:914-922

18. Wiltshire S, Hattersley AT, Hitman GA et al. (2001) A genomewide scan for loci predisposing to type 2 diabetes in a U.K. population (the Diabetes UK Warren 2 Repository): analysis of 573 pedigrees provides independent replication of a susceptibility locus on chromosome 1q. Am J Hum Genet 69:553-569

19. Vionnet N, Hani El-H, Dupont S et al. (2000) Genomewide search for type 2 diabetes-susceptibility genes in French whites: evidence for a novel susceptibility locus for earlyonset diabetes on chromosome 3q27-qter and independent replication of a type 2-diabetes locus on chromosome 1q21-q24. Am J Hum Genet 67:1470-1480

20. Ghosh S, Watanabe RM, Valle TT et al. (2000) The Finland-United States investigation of non-insulin-dependent diabetes mellitus genetics (FUSION) study. I. An autosomal genome scan for genes that predispose to type 2 diabetes. Am J Hum Genet 67:1174-1185

21. Duggirala R, Blangero J, Almasy et al. (1999) Linkage of type 2 diabetes mellitus and of age at onset to a genetic location on chromosome 10q in Mexican Americans. Am J Hum Genet 64:1127-1140

22. McCarthy MI (2003) Growing evidence for diabetes susceptibility genes from genome scan data. Curr Diab Rep 3:159-167

23. Frayling TM, Walker M, McCarthy MI et al. (1999) Parent-offspring trios: a resource to facilitate the identification of type 2 diabetes genes. Diabetes 48:2475-2479

24. Minton J, Hattersley AT, Owen KR et al. (2002) Association studies of genetic variation in the WFS1 gene and type 2 diabetes in UK populations. Diabetes 51:1287-1290 
25. Owen KR, Stride A, Ellard S, Hattersley AT (2003) Etiological investigation of diabetes in young adults presenting with apparent type 2 diabetes. Diabetes Care 26:2088-2093

26. Ewing B, Hillier L, Wendl MC, Green P (1998) Base-calling of automated sequencer traces using phred. I. Accuracy assessment. Genome Res 8:175-185

27. Ewing B, Green P (1998) Base-calling of automated sequencer traces using phred. II. Error probabilities. Genome Res 8:186-194

28. Nickerson DA, Tobe VO, Taylor SL (1997) PolyPhred: automating the detection and genotyping of single nucleotide substitutions using fluorescence-based resequencing. Nucleic Acids Res 25:2745-2751

29. Carlson CS, Eberle MA, Rieder MJ, Yi Q, Kruglyak L, Nickerson DA (2004) Selecting a maximally informative set of single-nucleotide polymorphisms for association analyses using linkage disequilibrium. Am J Hum Genet $74: 106-120$

30. Ye S, Dhillon S, Ke X, Collins AR, Day IN (2001) An efficient procedure for genotyping single nucleotide polymorphisms. Nucleic Acids Res 29:E88

31. Bengra C, Mifflin TE, Khripin Y et al. (2002) Genotyping of essential hypertension single-nucleotide polymorphisms by a homogeneous PCR method with universal energy transfer primers. Clin Chem 48:2131-2140

32. Abecasis GR, Cookson WO (2000) GOLD—graphical overview of linkage disequilibrium. Bioinformatics 16: $182-183$

33. Li C, Scott LJ, Boehnke M (2004) Assessing whether an allele can account in part for a linkage signal: the Geno-
type-IBD Sharing Test (GIST). Am J Hum Genet 74: 418-431

34. Gudbjartsson DF, Jonasson K, Frigge ML, Kong A (2000) Allegro, a new computer program for multipoint linkage analysis. Nat Genet 25:12-13

35. Zaykin DV, Westfall PH, Young SS, Karnoub MA, Wagner MJ, Ehm MG (2002) Testing association of statistically inferred haplotypes with discrete and continuous traits in samples of unrelated individuals. Hum Hered 53:79-91

36. Zhang L, Ge L, Tran T, Stenn K, Prouty SM (2001) Isolation and characterization of the human stearoyl-CoA desaturase gene promoter: requirement of a conserved CCAAT cis-element. Biochem J 357:183-193

37. Gabriel SB, Schaffner SF, Nguyen H et al. (2002) The structure of haplotype blocks in the human genome. Science 296:2225-2229

38. Ovcharenko I, Nobrega MA, Loots GG, Stubbs L (2004) ECR Browser: a tool for visualizing and accessing data from comparisons of multiple vertebrate genomes, Nucleic Acids Res 32 (Web Server Issue):W280-W286

39. Risch N, Teng J (1998) The relative power of family-based and case-control designs for linkage disequilibrium studies of complex human diseases I DNA pooling. Genome Res 8:1273-1288

40. Fingerlin TL, Boehnke M, Abecasis GR (2004) Increasing the power and efficiency of disease-marker case-control association studies through use of allele-sharing information. Am J Hum Genet 74:432-443 